\title{
CLASSIFICAÇÃO LAPAROSCÓPICA DA APENDICITE AGUDA. CORRELAÇÃO ENTRE GRAUS DA DOENÇA E AS VARIÁVEIS PERIOPERATÓRIAS
}

\author{
ACUTE APPENDICITIS LAPAROSCOPIC CLASSIFICATION. CORRELATION BETWEEN \\ DISEASE GRADE AND INTRAOPERATIVE VARIABLES
}

Carlos Augusto Gomes, TCBC-MG'; Tarcizo Afonso Nunes $^{2}$

\begin{abstract}
RESUMO: Objetivo: Apresentar a classificação laparoscópica da apendicite aguda e verificar a relação entre os graus da doença com o tempo de sintomas, tempo operatório, permanência hospitalar, complicações infecciosas e uso de antimicrobianos. Método: Estudo prospectivo, transversal, envolvendo 105 pacientes com diagnóstico de apendicite aguda e submetidos a apendicectomia laparoscópica entre Janeiro de 2000 e Julho de 2001. A doença foi classificada em grau 0 - Normal; 1 - Hiperemia e edema; 2 Exsudato fibrinoso; 3 - Necrose segmentar; 4A - Abscesso; 4B - Peritonite regional; 4C - Necrose da base do apêndice; 5 - Peritonite difusa. Resultados: A distribuição dos pacientes segundo a classificação foi: grau 0 (10,4\%); 1 (40\%); $2(29,5 \%) ; 3(2,9 \%) ; 4 \mathrm{~A}$ $(1,9 \%) ; 4 \mathrm{~B}(4,8 \%)$; 4C $(3,8 \%)$ e 5 (6,7\%). O tempo médio de início de sintomas acima de $40 \mathrm{~h}$ correlacionou-se com possibilidade de necrose e peritonite. O tempo operatório variou de 18 a 126 minutos, média de 31,4 minutos. A permanência hospitalar variou de 12 a 192 h, média de 39,5 h. A maior incidência de complicações Infecciosa ocorreu nos graus 4 e 5. O antimicrobiano foi de uso profilático graus 0,1 e 2 e terapêutico nos demais. A laparotomia foi necessária duas $(1,9 \%)$ vezes e não houve óbito. Conclusão: A classificação laparoscópica da apendicite aguda contemplou todas as formas clínicas da doença, possibilitou correlação com os tempos início de sintomas, operatório e de permanência hospitalar. Permitiu ainda, prever complicações infecciosas e racionalizar o uso de antimicrobianos (Rev. Col. Bras. Cir. 2006; 33(5): 289-293).
\end{abstract}

Descritores: Apendicite; Doença aguda; Laparoscopia/ classificação; Apendicectomia.

\section{INTRODUÇÂO}

A laparoscopia tem sido confirmada como abordagem segura e eficaz no diagnóstico e tratamento de doenças abdominais agudas ${ }^{1-3}$. Realizada pela primeira vez em 1981, por Kurt Semm ${ }^{4}$ a apendicectomia laparoscópica tornou-se uma opção atraente e alternativa à laparotomia no tratamento da apendicite aguda ${ }^{4}$. Estudos que comparam apendicectomia laparoscópica com laparotômica demonstram resultados favoráveis à primeira, sobretudo com relação à redução da dor pós-operatória, menores taxas de infecção do sítio cirúrgico, melhor resultado estético e retorno mais rápido ao trabalho ${ }^{5-9}$.

Nos últimos anos, o tratamento cirúrgico da apendicite aguda sofreu poucas modificações. As complicações pósoperatórias permaneceram em torno de $10 \%$, sendo a infecção do sítio cirúrgico responsável por um terço delas e com repercussões mais graves quando se empregam laparotomias amplas ${ }^{10}$. Assim, destaca-se a importância da abordagem laparoscópica, também como alternativa para reduzir as complicações e seqüelas ${ }^{11,12}$.

A classificação de doenças de acordo com estágio de evolução é importante para avaliar a gravidade e o prognóstico, além de permitir a elaboração de protocolos de orientação terapêutica e de pesquisa. A laparoscopia possi- bilitou visão ampla da cavidade abdominal e do apêndice, contribuindo para o inventário operatório. Proporcionou imagens "in situ" de vísceras, processos inflamatórios e secreções purulentas com mínimos traumatismos. Foi capaz de identificar as fases clínico-cirúrgicas da apendicite aguda, com base no processo inflamatório que envolve o apêndice ou toda a cavidade abdominal ${ }^{11,13,14}$. Observa-se que as classificações da apendicite aguda utilizadas, tanto para o procedimento laparotômico ${ }^{8,15-18}$, quanto para o laparoscópico ${ }^{5,19,20}$, permitem a adição de novos subgrupos representativos da abordagem em estudo, contribuindo na proposição de classificação mais abrangente.

O objetivo da pesquisa é apresentar classificação laparoscópica da apendicite aguda e verificar as relações entre os graus da doença com o tempo de início de sintomas, tempo operatório, permanência hospitalar, complicações infecciosas e uso de antimicrobianos.

\section{MÉTODO}

Após aprovação pela Comissão de Ética e Pesquisa do Hospital Monte Sinai de Juiz de Fora, Minas Gerais, estudou-se prospectivamente 105 pacientes com diagnóstico de apendicite aguda e submetidos a apendicectomia laparoscópica entre janeiro de 2000 a julho de 2001, pela equi-

1. Professor Assistente Mestre do Departamento de Cirurgia da Universidade Federal de Juiz de Fora (UFJF), Minas Gerais - Brasil.

2. Professor Adjunto Doutor do Departamento de Cirurgia da Universidade Federal de Minas Gerais (UFMG) - Brasil.

Recebido em 08-04-2006

Aceito para publicação em 07-05-2006

Conflito de interesses: nenhum

Fonte de financiamento: nenhuma

Dissertação apresentada ao Programa de Pós-graduação em Cirurgia da Faculdade de Medicina da Universidade Federal de Minas Gerais (UFMG) para obtenção do título de Mestre. 
pe de Cirurgia Digestiva da referida instituição. A idade variou de 13 a 74 anos, média de 30,8 anos, sendo $56,2 \%$ pacientes femininos e $88,6 \%$ leucodérmicos. Foram excluídos os pacientes menores de 12 anos, grávidas, portadores de acentuada distensão abdominal, instabilidade hemodinâmica e distúrbios da coagulação. O diagnóstico da doença foi obtido por meios clínicos, laboratoriais e ultra-sonografia. O tempo de sintomas da doença era registrado no protocolo por ocasião da consulta.

Todos os pacientes receberam anestesia geral e antibioticoterapia perioperatória com $2 \mathrm{~g}$ amoxacilinaclavulanato. Efetuou-se três vias de acesso à cavidade abdominal: uma no umbigo, para introdução do trocarte de 11 mm e a óptica Karl Storz (23006 BA) ${ }^{\circledR}$ de 30 graus, e duas outras, na região suprapúbica $(5 \mathrm{~mm})$ e na fossa ilíaca esquerda $(11 \mathrm{~mm})$. O paciente foi mantido na posição de "Trendelenburg" e decúbito lateral esquerdo. A exploração laparoscópica da cavidade abdominal que começou no hipocôndrio direito avaliou: cúpulas diafragmáticas ? hipocôndrio esquerdo ? flanco esquerdo ? pelve ? flanco direito ? íleo terminal. A seguir, procedia-se a classificação dos pacientes em graus, segundo os achados inflamatórios encontrados (Tabela 1).

Após a preensão e tração do apêndice o mesoapêndice era seccionado com eletrocoagulação. $\mathrm{O}$ tratamento do coto apendicular constou de posicionamento de dois clipes T 400 proximais e um distal, seguido de diérese com tesoura. Na presença de necrose da base do apêndice realizou-se a cecorrafia mediante laparotomia ou vídeo-assistida. O apêndice foi removido através do umbigo, protegido pelo trocarte ou em dispositivo coletor. A drenagem da cavidade abdominal foi empregada nos pacientes classificados como graus $4 \mathrm{~A}$ e $4 \mathrm{C}$. A laparotomia foi utilizada diante de dificuldades intra-operatórias de manipulação do processo inflamatório. Todos os apêndices foram encaminhados para estudo anatomopatológico.

O tempo operatório foi aferido em minutos a partir do início da operação até a última sutura. A permanência hospitalar foi registrada em horas. Considerou-se infecção do sítio operatório a drenagem de secreção purulenta até 30 dias de pós-operatório. As coleções intra-abdominais foram diagnosticadas por meio da ultra-sonografia. Os testes $t$ de student, análise de variância (ANOVA One - Way) e teste de Scheffè, verificaram a relação entre os graus da apendicite aguda com as variáveis selecionadas. Foi utilizado um nível de significância de 5\% $(\mathrm{p}<0,05)$. Empregou-se o software SPSS para o teste de Scheffé e o Microsoft Excel para os demais.

\section{RESULTADOS}

A distribuição dos pacientes operados segundo a classificação proposta.

Não foram evidenciadas alterações anatomopatológicas em 10,4\% dos apêndices examinados, 40\% foram classificados com grau 1 e $29,5 \%$ como grau 2 . Não houve diferença significativa entre os graus $3,4 \mathrm{~A}, 4 \mathrm{~B}, 4 \mathrm{C}$ e 5 (Tabela 2).
Tabela 1 - Classificação laparoscópica da apendicite aguda em graus, de acordo com os achados inflamatórios.

\begin{tabular}{ll}
\hline Grau & Achados Inflamatórios \\
\hline GRAU 0 & Normal \\
GRAU 1 & Hiperemia e edema \\
GRAU 2 & Exsudato fibrinoso \\
GRAU3 & Necrose segmentar \\
GRAU 4A & Abscesso \\
GRAU4B & Peritonite regional \\
GRAU4C & Necrose da base do apêndice \\
GRAU 5 & Peritonite difusa \\
\hline
\end{tabular}

Tabela 2 - Distribuição dos pacientes submetidos a apendicectomia laparoscópica de acordo com a classificação proposta.

\begin{tabular}{lrc}
\hline Grau & n & \% \\
\hline Grau 0 & 11 & $10,4 \% *$ \\
Grau 1 & 42 & $40,0 \% *$ \\
Grau 2 & 31 & $29,5 \% *$ \\
Grau 3 & 3 & $2,9 \%$ \\
Grau 4A & 2 & $1,9 \%$ \\
Grau 4B & 5 & $4,8 \%$ \\
Grau 4C & 4 & $3,8 \%$ \\
Grau 5 & 7 & $6,7 \%$ \\
Total & 105 & $100,0 \%$ \\
\hline
\end{tabular}

Nota: (*) teste t-Student $(p<0,05)$.

\section{antimicrobianos.}

Relação entre os graus da apendicite aguda e uso de

Todos receberam antibioticoterapia perioperatória com amoxacilina-clavulanato ( $2 \mathrm{~g})$ na indução anestésica. O antimicrobiano foi mantido por no máximo $24 \mathrm{~h}$ em 84 pacientes (80\%), classificados nos graus 0,1 e 2 . Naqueles 21 pacientes (20\%), classificados nos graus 3, 4 e 5, foram empregados ceftriaxona ( $2 \mathrm{~g} / \mathrm{dia}$ ) e metronidazol (1,5 g/ dia) por via venosa, com finalidade terapêutica por 5 a 10 dias, média de 7,38 $\pm 2,6$ dias.

Relação entre o tempo médio de sintomas da doença e os graus da apendicite aguda.

Houve diferença no tempo médio de sintomas da apendicite aguda, entre os graus 1 e $2(p<0,0001)$ e entre o grau 2 e os demais $(p<0,0005)$. Desenhou-se, portanto, três patamares contendo graus com tempos semelhantes (primeiro patamar: graus 0 e 1), (segundo: grau 2 isolado), (terceiro: graus 3, 4A, 4B, 4C e 5). (Figura 1).

\section{Relação entre o tempo médio operatório e os graus da apendicite aguda. \\ O tempo médio operatório de acordo com os graus} da apendicite aguda foi agrupado em patamares com tempos semelhantes. Os graus 1 e 2 constituíram o primeiro patamar ( $p$ 
$=0,988)$. Os graus 0,3 e 4A, o segundo $(\mathrm{p}=0,736)$. Os graus $4 \mathrm{~B}$ e 4 C representam o último $(p=0,843)$. Houve aumento significativo ( $\mathrm{p}<0,0001)$ do tempo médio operatório entre os patamares estabelecidos. $\mathrm{O}$ grau 5 apresentou tempo médio operatório superior à todos $(\mathrm{p}<0,0001)$ (Figura 2).

\section{Relação entre a permanência média hospitalar e os graus da apendicite aguda.}

A permanência média hospitalar de acordo com os graus da apendicite aguda foi também agrupada em patamares. Os graus 0 e 1 com médias semelhantes ( $p=0,998$ ), formaram o primeiro patamar. Os graus 2 e 3 e $4 \mathrm{~A}(p=0,998)$ constituíram o segundo. Da mesma forma, houve aumento significativo destes tempos $(p<0,0001)$ entre os patamares estabelecidos. A permanência média hospitalar dos graus $4 \mathrm{~B}, 4 \mathrm{C}$ e cinco diferiram entre si e entre os patamares $(p<0,0001)$ (Figura 3 ).

Incidência de complicações infecciosas de acordo com o grau da apendicite aguda.

A infecção do sítio operatório foi a complicação infecciosa mais freqüente, diagnosticada em sete $(6,7 \%)$ pacientes, sendo três do grau 2; um do grau 4B; dois do grau $4 \mathrm{C}$ e um do grau 5. Quatro $(3,7 \%)$ pacientes apresentaram coleção intraabdominal, sendo um do grau $4 \mathrm{~B}$ e três do grau 5 .

\section{DISCUSSÃO}

A laparoscopia avançou como método de escolha no tratamento da apendicite aguda e de outras causas de abdome agudo, após experiência adquirida com a realização dos procedimentos laparoscópicos eletivos e propedêuticos ${ }^{7,13,21}$.

A classificação da apendicite aguda em graus partiu da riqueza de informações obtidas com a laparoscopia na exploração da cavidade abdominal. Os fenômenos constituintes do processo inflamatório foram definidos de forma clara e concisa, no processo de graduação ${ }^{21}$. Preocupou-se, também, em sistematizar o tratamento operatório, além de avaliar a possibilidade do desenvolvimento de complicações infecciosas pósoperatórias.

Todos os pacientes receberam antibioticoterapia perioperatória com amoxacilina-clavulanato $(2 \mathrm{~g})$ na indução anestésica. Os antimicrobianos apresentam atividade contra germes aeróbicos gram negativos e anaeróbios. Nas operações potencialmente contaminadas (graus $0,1,2$ ), o antimicrobiano foi suspenso até 24 horas. Nas infectadas (graus 3, 4 e 5), o esquema de ceftriaxona e metronidazol foi utilizado por 5 a 10 dias. Desta forma, preservou-se os antimicrobianos de largo espectro para uso terapêutico, utilizando-se dos de espectro menos estendido para a profilaxia. É crescente, a prescrição de dose única para profilaxia antimicrobiana, entretanto esta conduta não foi padronizada na pesquisa ${ }^{22}$.

Em relação a distribuição dos pacientes de acordo com a classificação proposta, aqueles pacientes classificados nos graus 0,1 e 2 , apresentaram condução peroperatória padronizada, evolução previsível e bom prognóstico. Corresponderam às formas de apendicite aguda não complicadas referidas em outras publicações ${ }^{8,19,23}$. Em contrapartida, os classificados nos graus $4 \mathrm{~A}, 4 \mathrm{~B}, 4 \mathrm{C}$ e 5 , registraram diversida-

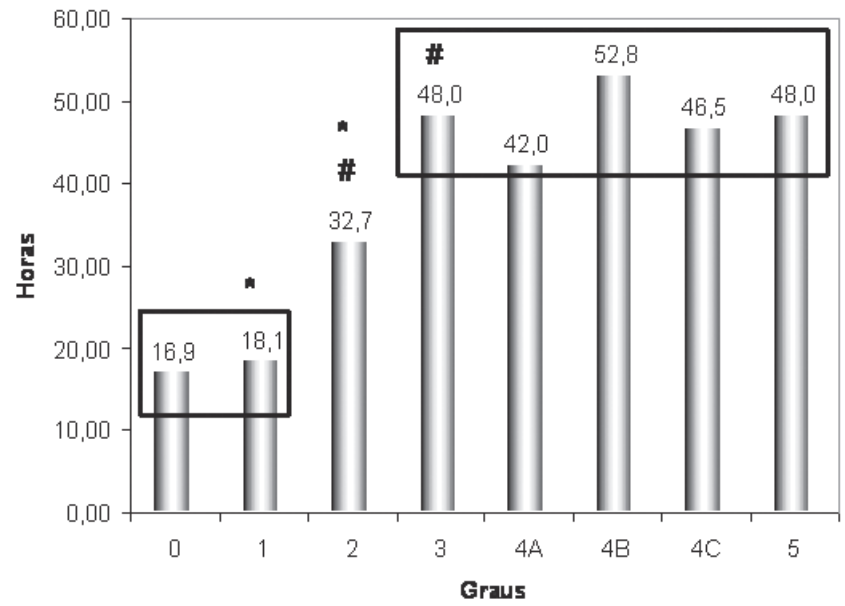

Figura 1 - Relação entre o tempo médio de sintomas da doença e o grau da apendicite aguda. $t$-Student: ( $\left.{ }^{*} p<0,0001\right)$; $(\# p<0,0005)$.

Tamanho: $127 \times 178 \mathrm{~mm}$

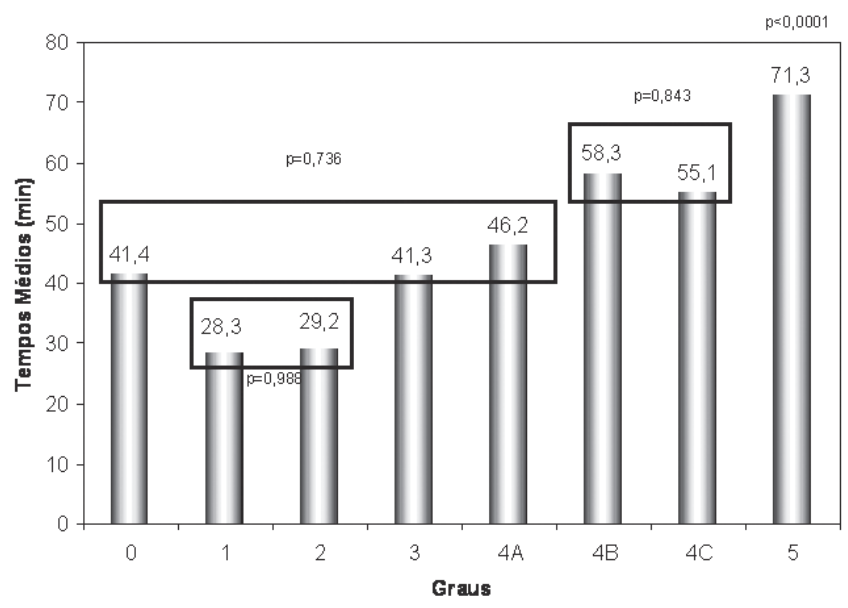

Figura 2 - Relação entre o tempo médio operatório e os graus da apendicite aguda. ANOVA e Scheffé $(p<0,0001)$.

Tamanho: $127 \mathrm{~mm} \times 178 \mathrm{~mm}$

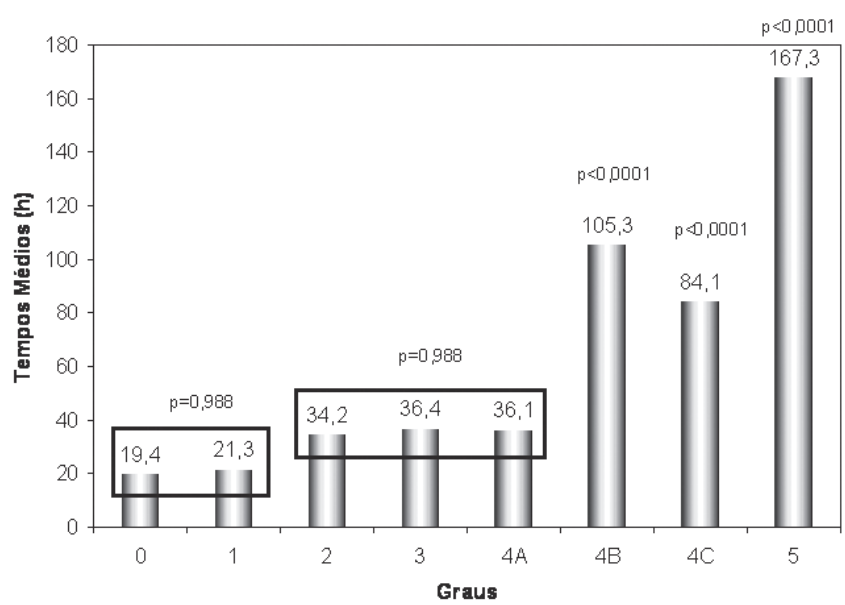

Figura 3 - Relação entre a permanência média hospitalar e os graus da apendicite aguda. ANOVA e Scheffé (Niveis $p<0,0001$ ).

Tamanho: $127 \mathrm{~mm} \times 178 \mathrm{~mm}$ 
des tanto de condutas intra-operatórias quanto dos resultados. Corresponderam às apendicites complicadas ou perfuradas de outras classificações ${ }^{8,19,23}$, que não exploraram tal estratificação na dimensão possibilitada pela laparoscopia. Os pacientes classificados no grau 3, apresentaram boa evolução pós-operatória a despeito da presença de necrose segmentar do apêndice.

A incidência de apêndice vermiforme com exame anatomopatológico normal (grau 0 ) foi de 10,4\%, valores dentro dos observados em outras referências ( $9 \%$ para o sexo masculino e $19 \%$ para o feminino $)^{24}$.

O tempo médio de sintomas da apendicite aguda, entre $40 \mathrm{~h}$ e $50 \mathrm{~h}$ relacionou-se com a possibilidade de necrose, abscesso e peritonite (Figura 1). Esses resultados são comparáveis aos da literatura, cujo tempo de início dos sintomas foi 2,5 vezes maior naqueles pacientes com apendicites agudas complicadas ${ }^{10,25}$. Ademais, pacientes com diagnóstico pós-operatório de apendicite aguda complicada, apresentaram retardo no atendimento hospitalar, duas vezes maior que quadros não complicados ${ }^{26}$.

A infecção do sítio operatório ocorreu em 6,7\% dos pacientes e foi a principal complicação pós-operatória. Ela é citada entre 0 a $15 \%$ para o procedimento laparotômico e entre 0 a $10 \%$ para o procedimento laparoscópico, e acreditase que a retirada do apêndice envolto por dispositivo coletor contribua para redução de sua freqüência ${ }^{9,27}$. Coleções de líquidos intra-abdominais foram diagnosticadas em quatro pacientes $(3,7 \%)$, que se submeteram à drenagem percutânea guiado pelo ultra-som. Somente em dois $(1,9 \%)$ casos foi confirmada a presença de pus. A freqüência de abscessos intra-abdominais após apendicectomia laparoscópica tem sido relatada entre 0 e $28 \%$ 9,14,28,29. Dentre os fatores considerados de risco para o desenvolvimento da complicação destacam-se a apendicite aguda gangrenada ou perfurada, e a disseminação de material infectado durante irrigação da cavidade abdominal ${ }^{29,30}$. Dentre os cuidados para redução dos abscessos intra-abdominais incluem-se: implementação de serviços de cirurgia laparoscópica, dissecção e exposição da base apendicular sem deixar resíduos ou lacunas, uso de dispositivos coletores, inspeção e sucção de líquidos no fundo de saco peritoneal ${ }^{28,29}$.

O tempo operatório e a permanência hospitalar apresentaram comportamento semelhante, aumentaram à medida que pacientes foram classificados em graus sucessivamente mais elevados (Figuras 2 e 3). O tempo médio operatório de 31,40 minutos, aqui observado, situou-se dentro dos publicados na literatura, verificados entre 20 e 43 minutos, para apendicite aguda não complicada ${ }^{31,32}$, e 81 a 114 minutos, para complicada ${ }^{14,21}$. O maior tempo médio operatório observado nos pacientes classificados no grau 0 , comparativamente aos graus 1 e 2, decorreu da necessidade de nova exploração da cavidade abdominal, à procura de outras afecções abdominais, que justificasse o diagnóstico clínico de abdome agudo. A permanência hospitalar variou de 12 a 192 h, média de 39,5 h. O tempo é inferior aos observados em outros trabalhos, situados entre 48 e $52,8 \mathrm{~h}^{8,33}$.

Assim, embora o número de pacientes classificados nos graus 3, 4 e 5 seja pequeno, verifica-se relação entre a classificação proposta e o comportamento das variáveis estudadas. Os graus 4 e 5 apresentaram maiores tempos médios e maiores possibilidades de complicações infecciosas. Neste aspecto a classificação contribuiu também, para avaliação da gravidade e prognóstico da doença.

A laparotomia foi necessária em dois (1,9\%) pacientes classificados como $4 \mathrm{C}$ e 5 . No primeiro foi realizada a cecorrafia vídeo-assistida, devido a necrose da base apendicular e, no segundo, foi empregada a laparotomia longitudinal mediana para a dissecção do acentuado processo inflamatório. A introdução do grau 4C (necrose da base) à classificação, é oportuna pelas dificuldades adicionadas ao tratamento laparoscópico do coto apendicular por meio de posicionamento de clipes. A cecorrafia laparoscópica, embora seja alternativa atraente, não foi realizada nesta pesquisa. $\mathrm{O}$ uso de grampeadores endoscópicos não foi usado em virtude do elevado custo.

Portanto, a classificação laparoscópica da apendicite aguda contemplou todas as fases de evolução da doença. Relacionou os graus da doença com tempo de sintomas, tempo operatório e a permanência hospitalar. Permitiu ainda, prever o desenvolvimento de complicações infecciosas pós-operatórias e racionalizar o uso de antimicrobianos. Paralelamente abre caminho para novas pesquisas, tanto no sentido de validar as conclusões, quanto avaliar protocolos terapêuticos de pacientes classificados em graus semelhantes.

\footnotetext{
ABSTRACT

Background: Laparoscopic classification of acute appendicitis is presented. The relationship between the grades of the disease with the time of symptoms, operating time, length of hospital stay, infectious complication rate and antimicrobials use is verified. Methods: Consecutive patients $(n=105)$ who underwent a laparoscopic appendectomy from January 2000, through July 2001, were studied in a prospective way. A classification of the disease in five grade was proposed: grade 0-Normal; 1 - Hyperemia and edema; 2 -Fibrinous exsudato; 3 -Segmentary necrosis; $4 A$-Abscess; $4 B$-Regional peritonitis; 4C-Appendix base necrosis; 5 -Diffuse Peritonitis. Results: The distribution of the patients according to classification was: grade 0 (10.4\%); 1 (40\%); 2 (29.5\%); 3 (2.9\%); $4 A$ (1.9\%); $4 B(4.8 \%) ; 4 C(3.8 \%)$ and 5 (6.7\%). The mean time of symptoms high than 40 hours was related with necrosis and peritonitis possibility. The mean operating time was 31,4 minutes (range $18-126$ minutes). The mean length of hospital stay was 39,5 hours (range 12 - 192 hours). The infectious complications were greater in grade 4 and 5. The antibiotics was used as prophylactic in grades 0,1 and 2 and therapeutically in the others. The conversion rate was $1.9 \%$ and there was no death. Conclusion: The laparoscopic classification of acute appendicitis contemplated all clinical forms of the disease, made possible correlation with the time of symptoms, operating time and length of hospital stay. It allowed also, to foresee infectious complications and to rationalize the antimicrobials.
}

Key words: Appendicitis; Acute disease; Laparoscopy/classification; Appendectomy. 


\section{REFERÊNCIAS}

1. Andersson RE, Hugander A, Ravn H, Offenbartl K, Ghazi SH, Nyström PO, Olaison G. Repeated clinical and laboratory examinations in patients with an equivocal diagnosis of appendicitis. World J Surg. 2000;24(4):479-85; discussion 485.

2. Barrat C, Catheline JM, Rizk N, Champault GG. Does laparoscopy reduce the incidence of unnecessary appendicectomies? Surg Laparoc Endosc. 1999;9(1):27-31.

3. Birnbaum BA, Wilson SR. Appendicitis at the millennium. Radiology. 2000;215(2):337-48.

4. Carbonell AM, Burns JM, Lincourt AE, Harold KL. Outcomes of laparoscopic versus open appendectomy. Am Surg. 2004;70(9):759-65; discussion 765-6.

5. Carr NJ. The pathology of acute appendicitis. Ann Diagn Pathol. 2000;4(1):46-58

6. Gangal TT, Gangal MH. Laparoscopic appendectomy. Endoscopy. 1987;19(3):127-9.

7. Gomes CA, Júnior CS, Oliveira DM, Cangussu IP, Peixoto RO, Júnior JRC. Utilização da videolaparoscopia no diagnóstico de abdome agudo. HU Rev. 2002;28(1/3):367-9.

8. Guzman-Valdivia GG. [An useful classification for acute appendicitis]. Rev Gastroenterol Mex. 2003;68(4):261-5.

9. Hellberg A, Rudberg C, Kullman E, Enochsson L, Fenyo G, Graffner H, Hallerback B, Johansson B, Anderberg B, Wenner J, Ringvist I, Sorensen S. Prospective randomized multicentre study of laparoscopic versus open appendicectomy. Br J Surg. 1999;86(1):48-53.

10. Johnson AB, Peetz ME. Laparoscopic appendectomy is an acceptable alternative for the treatment of perforated appendicitis. Surg Endosc. 1998;12(7):940-3.

11. Katkhouda N, Friedlander MH, Grant SW, Achanta KK, Essani R, Paik P, Velmahos G, Campos G, Mason R, Mavor E. Intraabdominal abscess rate after laparoscopic appendectomy. Am J Surg. 2000;180:456-60; discussion 460-1.

12. Kirshtein B, Roy-Shapira A, Lantsberg L, Mandel S, Avinoach E, Mizrahi S. The use of laparoscopy in abdominal emergencies. Surg Endosc. 2003;17(7):1118-24. Epub 2003 May 6.

13. Kraemer M, Ohmann C, Leppert R, Yang Q. Macroscopic assessment of the appendix at diagnostic laparoscopy is reliable. Surg Endosc. 2000;14(7):625-33.

14. Kum CK, Ngoi SS, Goh PM, Tekant Y, Isaac JR. Randomized controlled trial comparing laparoscopic and open appendicectomy. Br J Surg. 1993;80(12):1599-600.

15. Liu CC, Lu CL, Yen DH, Chern CH, Wang LM, Lee CH. Diagnosis of appendicitis in the ED: comparison of surgical and nonsurgical residents. Am J Emerg Med. 2001;19(2):109-12.

16. Martin LC, Puente I, Sosa JL, Bassin A, Breslaw R, McKenney MG, Ginzburg E, Sleeman D. Open versus laparoscopic appendectomy. A prospective randomized comparison. Ann Surg. 1995;222(3):256-61; discussion 261-2.

17. Majewski W. Diagnostic laparoscopy for the acute abdomen and trauma. Surg Endosc. 2000;14(10):930-7.

18. Moore DE, Speroff T, Grogan E, Poulose B, Holzman MD. Cost perspectives of laparoscopic and open appendectomy. Surg Endosc. 2005;19(3):374-8. Epub 2004 Dec 23.

19. Moberg AC, Ahlberg G, Leijonmarck CE, Montgomery A, Reiertsen O, Rosseland AR, Stoerksson R. Diagnostic laparoscopy in 1043 patients with suspected acute appendicitis. Eur J Surg. 1998;164(11):833-40; discussion 841.
20. Ng WT, Kong CH, Wong YT, Sze SY. Randomized clinical trial of laparoscopic versus open appendicectomy. Br J Surg. 2001;88(8):200-5.

21. Pereira Lima FE, Bogliolo L. Inflamações. In: Brasileiro-Filho G. Bogliolo patologia geral. $6^{\mathrm{a}}$ ed. Belo Horizonte: Guanabara Koogan; 2000. p. 112-48.

22. Pier A, Gotz F, Bacher C. Laparoscopic appendectomy in 625 cases: from innovation to routine. Surg Laparosc Endosc. 1991;1(1):8-13.

23. Piskun G, Kozik D, Rajpal S, Shaftan G, Fogler R. Comparison of laparoscopic, open, and converted appendectomy for perforated appendicitis. Surg Endosc. 2001;15(7):660-2. Epub 2001 May 14.

24. Reid RI, Dobbs BR, Frizelle FA. Risk factors for postappendicectomy intra-abdominal abscess. Aust N Z J Surg. 1999;69(5):373-4.

25. Sauerland S, Lefering R, Neugebauer EA. Laparoscopic versus open surgery for suspected appendicitis. Cochrane Database Syst Rev. 2004 (1):CD001546.

26. Semm K. Endoscopic appendectomy. Endoscopy. 1983;15(2):5964.

27. Slim K, Pezet D, Chipponi J. Laparoscopic or open appendectomy? Critical review of randomized, controlled trials. Dis Colon Rectum. 1998;41(3):398-403.

28. Smith DC. A historical overview of recognition of appendicitis. Part I. N Y State J Med. 1986; 85(11):571-83.

29. Tang E, Ortega AE, Anthone GJ, Berart RW Jr. Intraabdominal abscesses following laparoscopic and open appendectomies. Surg Endosc. 1996;10(3):327-8.

30. Temple CL, Huchcroft SA, Temple WJ. The natural history of appendicitis in adults. A prospective study. Ann Surg. 1995;221(3):279-81.

31. Weed HG. Antimicrobial prophylaxis in the surgical patients. Med Clin North Am. 2003;87(1):59-75.

32. Wilcox RT, Traverso LW. Have the evaluation and treatment of acute appendicitis changed with new technology? Surg Clin North Am. 1997;77(6):1355-70.

33. Wullstein C, Barkhausen S, Gross E. Results of laparoscopic vs. conventional appendectomy in complicated appendicitis. Dis Colon Rectum. 2001;44(11):1700-5.

34. Yao CC, Lin CS, Yang CC. Laparoscopic appendectomy for ruptured appendicitis. Surg Laparosc Endosc Percutan Tech. 1999;9(4):271-3.

Como citar este artigo:

Gomes CA, Nunes TA. Classificação laparoscópica da apendicite aguda. Correlação entre graus de doença e variáveis perioperatórias. Rev Col Bras Cir. [periódico na Internet] 2006 Set-Out;33(5). Disponível em URL; www.scielo.br/rcbc

Endereço para correspondência:

Carlos Augusto Gomes

Rua Sampaio n 346 / 801

36013-240 - Juiz de Fora - MG

FAX: + 55032 323232-2002.

E-mail: caxiao@fusoes.com.br 